Case Report

\title{
The Combination of Interferon-Alpha and Ponatinib Enables Faster and Deeper Molecular Responses in Patient with De Novo Blast Crisis of CML: Interferon-Alpha May Return as a CML Treatment
}

\author{
Kunio Hayashi $\mathbb{D}^{1},{ }^{1}$ Kazuhiro Ikegame, ${ }^{2}$ and Naoto Takahashi $\mathbb{D}^{3}$ \\ ${ }^{1}$ Division of Hematology, Meiwa General Hospital, 4-31 Agenaruocho, Nishinomiya, Hyogo 663-8186, Japan \\ ${ }^{2}$ Division of Hematology, Hyogo College of Medicine, Mukogawacho, Nishinomiya, Hyogo 663-8501, Japan \\ ${ }^{3}$ Department of Hematology Nephrology and Rheumatology, Akita University, 1-1 Hondo 1-Chome, Akita 010-8543, Japan
}

Correspondence should be addressed to Naoto Takahashi; naotot@doc.med.akita-u.ac.jp

Received 6 February 2021; Revised 31 March 2021; Accepted 16 April 2021; Published 4 May 2021

Academic Editor: Pier Paolo Piccaluga

Copyright $(92021$ Kunio Hayashi et al. This is an open access article distributed under the Creative Commons Attribution License, which permits unrestricted use, distribution, and reproduction in any medium, provided the original work is properly cited.

In the era of tyrosine kinase inhibitor (TKI) treatment, its effectiveness in treating chronic myelogenous leukemia (CML) has been improved, ensuring the same prognosis as that of healthy people of the same age. However, there are some patients with de novo blast crisis that undergoes acute conversion from the time of diagnosis and does not respond to TKI treatment, especially in the older patients. Here, we present a case of an older patient with de novo lymphoid crisis who was first treated with a combination of TKI and chemotherapy, but it was difficult to maintain a durable deep molecular response (DMR). After he achieved major molecular response (MMR) or less, it was possible to suppress IS\% to DMR by performing a combined treatment with interferon$\alpha($ IFN- $\alpha)$ and ponatinib. It is considered that DMR can be maintained by the combination of the two-way action of IFN- $\alpha$, that is, the transfer of dormant CML stem cells to the cellcycle and the activation of a specific immune response to CML cells. This clinical result suggests the possibility of the re-emergence of IFN- $\alpha$, which has been used a therapeutic drug in the past.

\section{Introduction}

Long-term survival in patients with chronic myelogenous leukemia (CML) was not possible to achieve until the introduction of tyrosine kinase inhibitor (TKI) therapy. Until the era of TKI treatment, allogeneic hematopoietic stem cell transplantation (allo-SCT) and interferon- $\alpha$ (IFN$\alpha)$ therapy were the available treatment options; however, they were successful only in a limited number of people. TKIs have significantly improved survival outcomes in CML patients [1], and the possibility to discontinue TKI treatment for successful cases without recurrence has been recently explored $[2,3]$. However, there are some CML patients who do not achieve a deep molecular response (DMR) and they have to continue TKI therapy in spite of various side effects. In the case of de novo blast crisis, it is much more difficult to achieve MRD [4]. Although allo-
SCT can be selected as a treatment mode, it is associated with high risks and is generally not indicated for older patients. For patients with chronic phase CML (CML-CP) who cannot obtain optimal response, some investigators were using a combination of IFN- $\alpha$ and TKI for treatment $[5,6]$. Through this combination treatment, they have succeeded in obtaining a durable DMR [7]. In this report, we present a case of a patient with de novo blast crisis treated with the same combination after the chemotherapy to obtain early DMR and maintain a stable condition. This combination of IFN- $\alpha$ and TKI suggests the possibility of a resurgence of IFN- $\alpha$ in the treatment of CML.

\section{Case Presentation}

A 68-year-old man was referred to our hospital, who presented with fever and loss of appetite. His routine annual 
TABle 1: Laboratory findings on admission.

Peripheral blood

WBC

Blast

Stab

Seg

Lymph

Mono

Eo

Baso

RBC

$\mathrm{Hb}$

$\mathrm{Ht}$

Plt

NAP-R

NAP-S

Coagulation

PT

APTT

Fibrinogen

FDP

D-dimer

AT-III

Biochemistry

TP

Alb

Tbili

Dbili

AST

ALT

$\mathrm{LDH}$

$\gamma$ GTP

BUN

$\mathrm{Cr}$

UA

eGFR

Glu

CRP

Ferritin

Bone marrow

NCC

Mgk

Blast

$\mathrm{M} / \mathrm{E}$

$G$-Band karyotype of bone marrow

$46, \mathrm{XY}, \mathrm{t}(9 ; 22)(\mathrm{q} 34 ; \mathrm{q} 11.2)[16]$

46, XY [4]

$B C R-A B L 1$ transcripts

Major BCR-ABL1 $18 \times 10 e 5$, HLA-DR+

Immunophenotype of blasts in bone marrow

$\mathrm{CD} 34+, \mathrm{HLA}=\mathrm{DR}+, \mathrm{MPO}-, \mathrm{CD} 3-, \mathrm{TdT}+, \mathrm{CD} 19+, \mathrm{CD} 79 \mathrm{a}+, \mathrm{CD} 13 \pm$

Abbreviation: WBC, white blood cells; blast, myeloblast; myelo, myelocytes; meta, metamyelocytes; stab, stab cells; seg, segmented cells; lym, lymphocytes; mono, monocytes; eo, eosinophiles; baso, basophiles; Hb, hemoglobin; Ht, hematocrit; Plt, platelet; NAP-rate, neutrophil alkaline phosphatase activities-rate; NAT-score, neutrophil alkaline phosphatase activities-score; NCC, nuclear cell counts; Mgk, megakaryocytes; M/E, myeloid erythroid ratio; PT, prothrombin time; APTT, activated partial thromboplastin time; FDP, fibrin/fibrinogen degradation product; AT-III, antithrombin-III.

health checkup results were normal and he had no history of an illness that required hospitalization. He had developed fever and cough approximately 10 days previously and had been treated by a doctor nearby. However, a blood test revealed a high white blood cell count of $25,000 / \mu \mathrm{l}$ and he was advised to visit our hospital. Laboratory test results on admission are shown in Table 1. Although he was suspected of having Philadelphia chromosome-positive acute lymphoid leukemia, the diagnosis of de novo lymphoid blast crisis CML was confirmed based on the results of the 


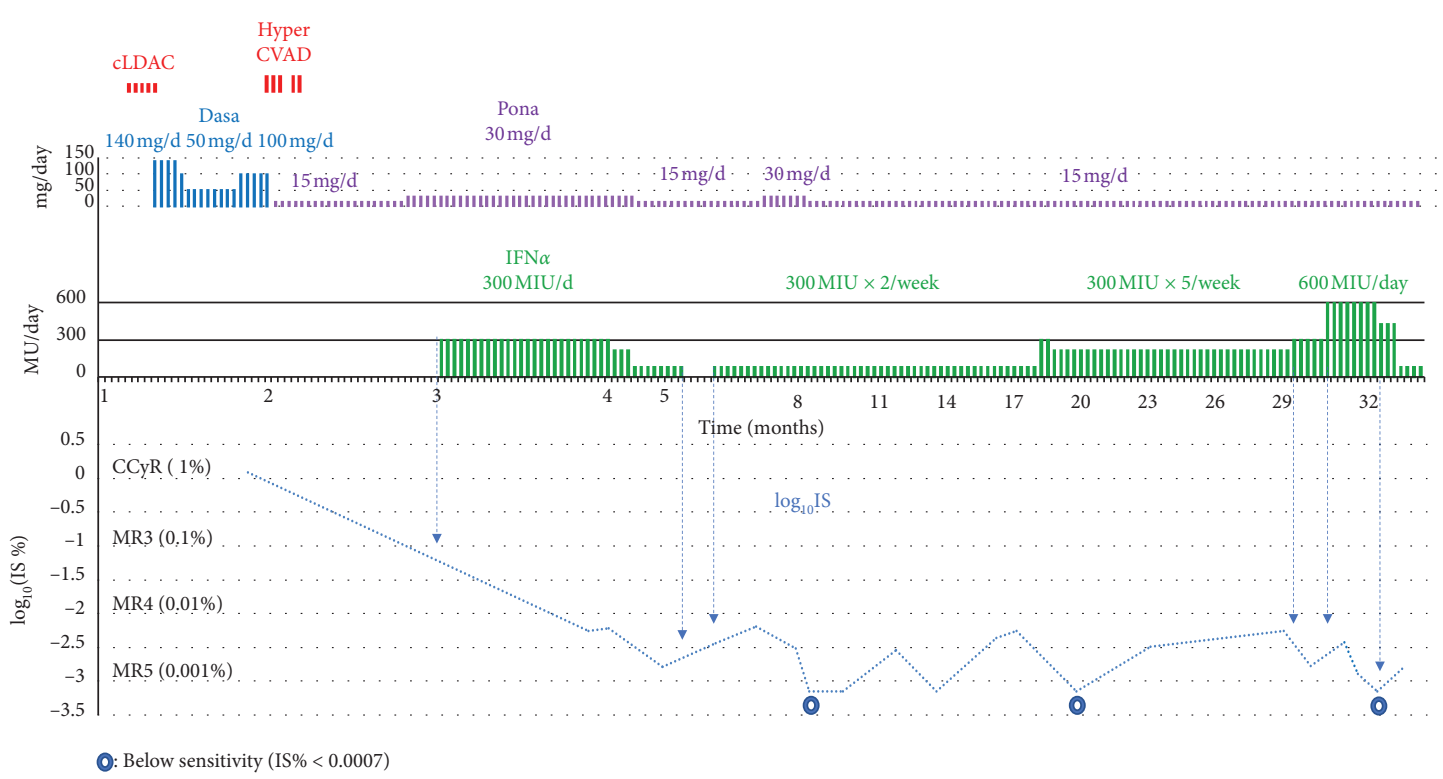

Figure 1: Clinical course of de novo blast crisis. Abbreviation: Nilo, nilotinib; Dasa, dasatinib; Pona, ponatinib; cLDAC, continuous infusion of low dose cytarabine (AraC); Hyper CVAD, hyperfractionated cyclophosphamide, vincristine, doxorubicin (adriacin), and dexamethasone.

examinations. In the immunophenotypic data, blasts strongly expressed HLA-DR, CD34, CD19, CD10, and CD79a. The breakpoint was found in the major BCR, and the minor breakpoint region associated with $\mathrm{Ph}$ chromosome positive acute lymphoblastic leukemia was not detected. Dasatinib was initiated as the first-line therapy with a continuous low-dose cytarabine, but there was no hematological effect. The chemotherapy regimen was changed to the reduced doses of cyclophosphamide, vincristine, doxorubicin (adriacin), and dexamethasone (Hyper CVAD) with ponatinib $15 \mathrm{mg} /$ day $[6,8]$. Hematological remission was obtained on day 40 of this treatment. At that time, the bone marrow nucleated cell count was $1.1 \times 10^{4} / \mu \mathrm{l}$ and the proportion of blast cells was $1.4 \%$, while the major BCRABL1 mRNA was $94 \times 10^{3}$ copies/ $\mu$ g. After the chemotherapy with Hyper CVAD, white blood cells were less than $1000 / \mu \mathrm{l}$ for 20 days and neutrocytes were less than $500 / \mu \mathrm{l}$ for 22 days during which time endogenous infection was complicated even under antibiotic treatment. In order to avoid worsening infectious diseases of febrile neutropenia, the chemotherapy was discontinued and treatment was changed to a combination of ponatinib $30 \mathrm{mg} /$ day and IFN$\alpha$ (300 MIU/day). In one month, the IS-PCR value was decreased to $0.0056 \%$ (MR4). The dose of ponatinib was reduced to $15 \mathrm{mg} /$ day and IFN- $\alpha$ was continued at $300 \mathrm{MIU} /$ day twice a week; after that, the IS-PCR value was decreased further to $0.0016 \%$. When IFN- $\alpha$ was discontinued, the ISPCR value was increased to $0.0064 \%$. When IFN- $\alpha 300 \mathrm{MIU} /$ day was restarted twice a week, the IS-PCR value was decreased to $0.0007 \%$ after 4.5 months and it fell below the detection sensitivity limit after another 4 months. The ISPCR value was not stable and was increased again to $0.0055 \%$ after 3 months. When the dose of IFN- $\alpha$ was increased to $300 \mathrm{MIU} /$ day five days a week, the IS-PCR value fell below the detection sensitivity in 2 months. Frequent measurements of the BCR-ABL1 transcript revealed fluctuations in its values, and IFN- $\alpha$ suppressed it and reduced the width of fluctuations in a dose-dependent manner. The course of treatment and the transition of IS\% are shown in Figure 1. Because the de novo blast crises in older patients are maladapted for stem cell transplantation, such a deep molecular genetic response of BCR-ABL1 to INF- $\alpha$ is a great way to obtain a better prognosis.

\section{Discussion}

In the present case of the de novo lymphoid crisis in older patient, we succeeded to suppress the fluctuations in IS values and maintain DMR using the treatment of ponatinib combined with IFN- $\alpha$. IFN- $\alpha$ therapy has become less commonly used with the advent of TKIs, but some studies suggested the possible effective use of IFN- $\alpha$. In the Stop Imatinib (STIM) study, which first investigated the discontinuation of TKIs, having a history of IFN- $\alpha$ treatment was one of the prognostic factors that enhanced treatmentfree response (TFR) [9]. The German CML and Scandinavian CML research groups have shown that a mode of combination treatment with IFN- $\alpha$ could elicit a faster and deeper response in high-risk CML patients than treatment with imatinib alone. These studies suggested that IFN- $\alpha$ provided clinical benefits [10].

TKIs have the effect of inactivating Ph-positive leukemia cells and inducing apoptosis but cannot eliminate these cells directly [3]. IFN- $\alpha$ acts on dormant leukemia stem cells, which cause the relapse, and recruits them into the cell cycle to be affected by TKI and further regulates gene expression in CML cells or induces cytotoxic T cells and natural killer cells [11]. Due to the synergistic effect of these various actions, the combined treatment of ponatinib and IFN- $\alpha$ is thought to suppress the progression of the disease. As 
observed in our case, the efficacy of IFN- $\alpha$ appears to be dose-dependent, but detailed studies to establish the optimal dosage and treatment duration of IFN- $\alpha$ are needed in the future.

\section{Data Availability}

The authors confirm that the data supporting the findings of this study are available.

\section{Consent}

IFN- $\alpha$ and ponatinib have been approved for the treatment of CML. Since no direct study of this combination treatment has been conducted, we obtained the approval of the ethics committee in our hospital and explained the need for this treatment to the patient and received his consent.

\section{Conflicts of Interest}

Kunio Hayashi and Kazuhiro Ikegame report no conflicts of interest regarding the content of this paper. Naoto Takahashi declares that he has received lecture fees and scholarship donations and conducted joint research with Otsuka Pharmaceutical.

\section{References}

[1] B. J. Druker, F. Guilhot, S. G. O’Brien et al., "Five-year followup of patients receiving imatinib for chronic myeloid leukemia," New England Journal of Medicine, vol. 355, no. 23, pp. 2408-2417, 2006.

[2] E. Jabbour, "Chronic myeloid leukemia: first-line drug of choice," American Journal of Hematology, vol. 91, no. 1, pp. 59-66, 2016.

[3] A. Hochhaus, M. Baccarani, R. T. Silver et al., "European leukemianet 2020 recommendations for treating chronic myeloid leukemia," Leukemia, vol. 34, no. 4, pp. 966-984, 2020.

[4] P. Jain, H. M. Kantarjian, A. Ghorab et al., "Prognostic factors and survival outcomes in patients with chronic myeloid leukemia in blast phase in the tyrosine kinase inhibitor era: cohort study of 477 patients," Cancer, vol. 123, no. 22, pp. 4391-4402, 2017.

[5] V. Polivkova, P. Rohon, H. Klamova et al., "Interferon- $\alpha$ revisited: individualized treatment management eased the selective pressure of tyrosine kinase inhibitors on BCR-ABL1 mutations resulting in a molecular response in high-risk CML patients," PLoS One, vol. 11, no. 5, Article ID e0155959, 2016.

[6] Y. Motomura, K. Arai, K. Yoshifuji et al., "Chronic myeloid leukemia harboring T315I and F317L mutations successfully treated with interferon- $\alpha$ and ponatinib," Rinsho Ketsueki, vol. 60, no. 1, pp. 33-38, 2019.

[7] C. Cumbo, L. Anelli, G. Specchia, and F. Albano, "Monitoring of minimal residual disease (MRD) in chronic myeloid leukemia: recent advances," Cancer Management and Research, vol. 12, pp. 3175-3189, 2020.

[8] K. Sasaki, E. J. Jabbour, F. Ravandi et al., "Hyper-CVAD plus ponatinib versus hyper-CVAD plus dasatinib as frontline therapy for patients with Philadelphia chromosome-positive acute lymphoblastic leukemia: a propensity score analysis," Cancer, vol. 122, no. 23, pp. 3650-3656, 2016.
[9] F.-X. Mahon, D. Réa, J. Guilhot et al., "Discontinuation of imatinib in patients with chronic myeloid leukaemia who have maintained complete molecular remission for at least 2 years: the prospective, multicentre stop imatinib (STIM) trial," The Lancet Oncology, vol. 11, no. 11, pp. 1029-1035, 2010.

[10] P. E. Westerweel and P. A. W. te Boekhorst, "New approaches and treatment combinations for the management of chronic myeloid leukemia," Frontiers in Oncology, vol. 9, pp. 1-7, 2019.

[11] M. Talpaz, R. Hehlmann, A. Quintás-Cardama, J. Mercer, and J. Cortes, "Re-emergence of interferon- $\alpha$ in the treatment of chronic myeloid leukemia," Leukemia, vol. 27, no. 4, pp. 803-812, 2013. 